\title{
CICLO DE VIDA E ECOLOGIA DE Paspalum fasciculatum WILLD. EX. FLUEGGE (POACEAE), NA VÁRZEA DA AMAZÔNIA CENTRAL
}

\author{
Auristela dos Santos CONSERVA ${ }^{1}$, Maria Teresa Fernandez PIEDADE ${ }^{2}$
}

Resumo - Paspalum fasciculatum é uma robusta gramínea $\mathrm{C}_{4}$, perene, de ocorrência nas várzeas da Amazônia Central. Para estabelecer a ecologia e o ciclo de vida da espécie em relação as flutuaçōes anuais do nivel do rio, vinte plantas foram marcadas ao acaso e acompanhadas mensalmente, de setembro/95 a agosto /96, em duas áreas entre 23 e 27 m (s.n.m.). Registrouse o comprimento do talo, o número de folhas verdes e mortas, brotos, e a presença de inflorescências. Paspalum fasciculatum sincroniza seu crescimento com a fase terrestre do ciclo hidrológico, tendo atingido no periodo de estudo o comprimento máximo de $4,53 \mathrm{~m}$. A dormência e grande tolerância dos talos a anos subsequentes de inundação e a capacidade de suportar mudanças bruscas do ambiente físico, permitem classificar a espécie como estrategista " $r$ ", altamente adaptada e representativa dos primeiros estágios de colonização das margens sedimentares de rios e lagos de várzea.

Palavras-chave: Paspalum fasciculatum, ciclo de vida, áreas inundáveis, várzea, Amazônia Central.

Life Cycle and Ecology of Paspalum fasciculatum Willd. ex. Fluegge (Poaceae), in the Vảrzea of the Central Amazon

\begin{abstract}
Paspalum fasciculatum is a robust perennial $\mathrm{C}_{4}$ grass that occurs in the Central Amazon várzeas. In order to establish the ecology and life cycle of the species in relation to the annual water level fluctuations, twenty plants were randomly selected and monthly observed from September $/ 95$ to August $/ 96$, in two sites from 23 to $27 \mathrm{~m}$ (a.s.l.). The length of stems, and the number of live and dead leaves, sprouts and inflorescence were recorded. The growth of Paspalum fasciculatum is synchronised with the terrestrial phase of the hydrological cycle, reaching during the period of the study a maximum length of $4.53 \mathrm{~m}$. The dormancy and ability of stems to survive subsequent years under flood, and the tolerance of the species to a highly unstable environment allow us to classify it as a extremely adapted " $r$ " strategist, representing the first stages of colonisation on the sedimentary margins of rivers and lakes in the várzea.
\end{abstract}

Key-words: Paspalum fasciculatum, life cycle, floodplains, várzea, Central Amazon.

\section{INTRODUÇÃO}

As planícies inundáveis, que na região Amazônica cobrem cerca de $300.000 \mathrm{~km}^{2}$ (Junk, 1993), são definidas como áreas periodicamente inundadas pelo transbordamento lateral de rios ou lagos, e/ou pela precipitação direta ou água subterrânea. Um rio e suas planícies inundáveis formam uma unidade ecológica que tem o pulso de inundação como principal força dirigente do sistema. As oscilações de nível da água provocam uma série de transformações nas características limnológicas dos corpos de água, determinando a estrutura de todos os biótopos aquáticos e semi-aquáticos dos principais sistemas de rios do vale

\footnotetext{
'Projeto INPA/MAX-PLANCK, Instituto Nacional de pesquisas da Amazônia (INPA) Curso

Biologia de Água Doce. caixa postal 478, 69011-970 Manaus - AM, Brasil.

${ }^{2}$ INPA/CPBA, Projeto INPA/MAX-PLANCK - Caixa postal 478, 69011-970 Manaus - AM, Brasil.
} 
do Amazonas (Junk et al,. 1989). Na Amazônia Central, a amplitude média anual da inundação é de $10 \mathrm{~m}$ (Schmidt, 1973).

As áreas de alagação da região amazônica são formadas por um complexo de rios, lagos, furos, paranás e pequenos igarapés, interconectados durante as águas altas, através do pulso de inundação. Conforme a natureza nutricional do rio associado à área alagável, podem ser definidos dois grandes grupos, os igapós, associados a áreas de águas claras ou negras, com $\mathrm{pH}$ ácido, e pobres em minerais, e as várzeas, áreas com $\mathrm{pH}$ neutro e com grande carga de minerais (Prance, 1979; Furch \& Junk, 1997). Essas diferenças relacionam-se à geologia da nascente e da área de captação dos rios (Sioli, 1975).

As comunidades bióticas mais características das várzeas são as macrófitas aquáticas (Junk, 1970; Sioli, 1984). Essa definição, bastante genérica, diz respeito às plantas crescendo na água, solos cobertos por água ou em solos geralmente saturados de água. $\mathrm{Na}$ Amazônia, muitas plantas podem ser classificadas como macrófitas aquáticas (Junk \& Howard-Williams, 1984). Apenas na várzea da Amazônia Central, nas cercanias da cidade de Manaus, foram identificadas 388 espécies de plantas herbáceas (Junk \& Piedade, 1993a, b). Entretanto, apesar do elevado número, apenas 5 espécies de gramíneas podem ser consideradas como dominantes, ocorrendo em grupamentos mono-específicos, ocupando grandes exten-sões, e grande parte delas apresentando grande produção anual de biomassa (Junk \& Piedade, 1993a; 1993b; 1997).

Assume-se que $10 \%$ dos sistemas de várzea, o que corresponde a $20.000 \mathrm{~km}^{2}$ (Junk, 1993), são cobertos por três das mais produtivas gramíneas da região, Echinochloa polystachya, Paspalum repens e Paspalum fasciculatum (Piedade et al., 1994). O ciclo de vida de $E$. polystachya já foi acompanhado (Piedade, 1993), como também o ciclo de vida de $P$. repens (Junk, 1970). Entretanto, ainda persiste essa lacuna no que diz respeito ao ciclo de vida de $P$. fasciculatum.

Paspalum fasciculatum parece ser uma espécie de grande exigência nutricional, uma vez que sua ocorrência é restrita às áreas de várzea (Martius, 1842; Index Kwensis, 1895). Além da natureza geológica dos rios associados, o regime de nutrientes é também influenciado pelas flutuações anuais de nível da água dos rios (Howard-Williams \& Junk, 1976; 1977; Junk \& Howard-Williams, 1984). A ciclagem de nutrientes e a transferência de energia são mediadoras das relações entre os ambientes aquáticos e terrestres das planícies inundáveis de várzea, sendo Paspalum fasciculatum um bom exemplo de planta adaptada a esses habitats.

Segundo Sioli (1985), para se entender as interações recíprocas que regem o funcionamento de um ambiente como um todo, a melhor base é começar pelo estudo das populações desses ambientes. Considerando que dentre as cinco 
espécies de gramíneas da várzea da Amazônia Central Paspalum fasciculatum Willd. Ex. Fluegge. é uma das mais importantes, devido à sua alta produção de biomassa (Junk \& Piedade, 1993b; 1997), e pelo fato do ciclo de vida e ecologia da espécie não haver sido estudado convenientemente, escolhemos essa espécie para desenvolver este trabalho.

Estudos que estabeleçam a ecologia e o ciclo de vida de espécies de importância funcional dentro de um ecossistema, tanto para o conhecimento científico, como para o fornecimento de subsídios para planos de manejo e uso racional de áreas de várzea, são fundamentais. Por outro lado, importantes variações no ciclo de vida das espécies podem ser verificadas de acordo com habitat no qual ela se estabelece dentro das áreas alagáveis. Por essas razões, este estudo tem como objetivo, estabelecer a ecologia e o ciclo de vida de $P$. fasciculatum, na margem de um lago de várzea e na margem do Rio Solimões, relacionando as variações observadas aos fatores abióticos predominantes.

\section{Descrição botânica da planta}

\section{Paspalum fasciculatum Willd.} Ex. Fluegge. é uma robusta gramínea $\mathrm{C}_{4}$, perene, com talos que podem alcançar um comprimento de mais de $4 \mathrm{~m}$. A folhas têm lâmina suberecta, linear, longe acuminata e glabra. As inflorescências são do tipo panícula composta. As espécies perenes deste gênero geralmente apresentam tufos de raizes fibrosas, ou rizomatosas
(Martius, 1842; Index Kwensis, 1895; Cook et al., 1974).

A espécie é um capim nativo de ocorrência espontânea nas áreas de várzea da Amazônia Central, tendo sido descrita em 1569 por Willdenow (Index Kwensis, 1895; Martius, 1842).

Na região Amazônica $P$. fasciculatum é popularmente chamada de "Muri" ou "Capim Mori", enquanto que em Goiás recebe o nome de "Capim do Araguaia", e no Mato Grosso "Capim da Praia" (Martius, 1842).

$\mathrm{O}$ gênero Paspalum possui mais de duzentas espécies, que se encontram distribuídas por toda a América Tropical, com poucas espécies no velho mundo. Na América do Sul, Paspalum fasciculatum tem sua ocorrência descrita no Japurá (Alto Amazonas), em Óbidos, em Santarém, em Montevidéu (Uruguai) e no Peru (Martius, 1842).

\section{Área de Estudo}

Segundo Junk (1970), na regiào Amazônica existem cinco principais habitats colonizados pelas macrófitas aquáticas: o canal principal do Rio Solimões/Amazonas; os bancos e regiões de sedimentação dos rios de água branca; lagos de várzea sujeitos às grandes flutuações de nivel da água; lagos de várzea sujeitos a uma flutuação relativamente pequena de nivel da água, e as águas pretas onde ocorrem poucas espécies ocasionalmente e em pequenas manchas.

Entre os cinco ambientes citados, foram escolhidos dois: os bancos e regiões de sedimentação dos 
rios de água branca, neste caso uma margem direita do Rio Solimões ( $3^{\circ}$ $14^{\prime} 44^{\prime \prime} \mathrm{S}$ e $59^{\circ} 53^{\prime} 36^{\prime \prime} \mathrm{W}$ ), chamada localmente de Curari, e um lago de várzea sujeito às grandes flutuações de nível da água, o Lago Central (3ำ $14^{\prime}$ $44^{\prime \prime}$ S e $59^{\circ} 57^{\prime} 47^{\prime \prime} \mathrm{W}$ ), localizado na Ilha da Marchantaria (Fig. 1).

O sitio amostral do Rio Solimões se localiza a aproximadamente $20 \mathrm{~km}$ da cidade de Manaus. Nele foi delimitada uma área de $3125 \mathrm{~m}^{2}$, situada na cota de $27 \mathrm{~m}$ sobre o nivel do mar (s.n.m.) (Fig. 2). A Ilha da Marchantaria, onde localizase o Lago Central, situa-se no Municipio de Iranduba, (3ำ $15^{\prime} \mathrm{S}$ e $60^{\circ}$ $00^{\prime} \mathrm{O}$ ), situando-se a cerca de $15 \mathrm{~km}$ da foz do Rio Negro e $20 \mathrm{~km}$ da cidade de Manaus (Fig. 1). Nas margens do Lago Central, em um sítio amostral localizado na cota de $23 \mathrm{~m}$ (s.n.m.), delimitou-se também uma área de $3125 \mathrm{~m}^{2}$ (Fig. 3).

O relevo do sítio amostral do Solimões era relativamente plano, e a

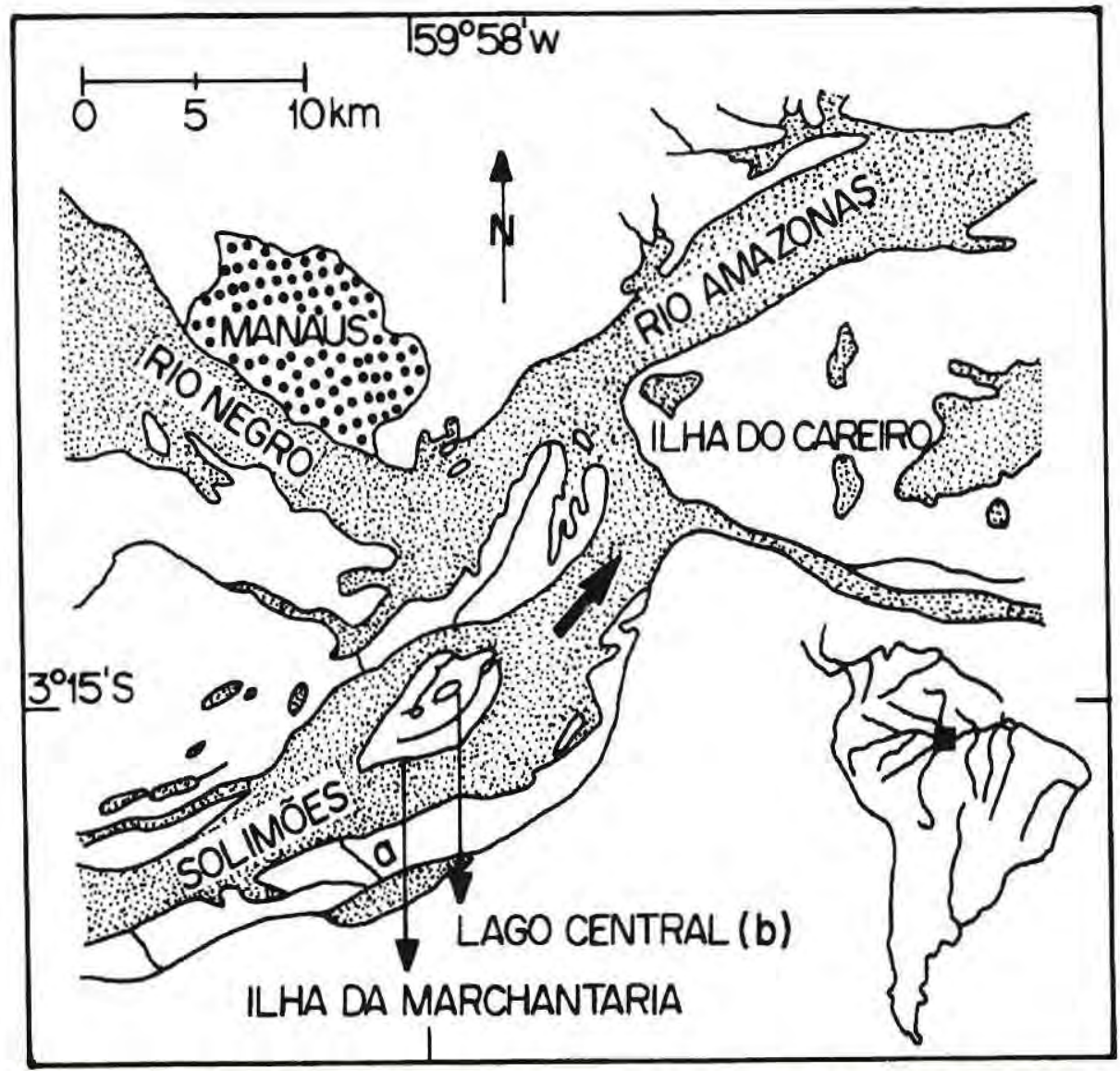

Figura 1. Mapa da localização das áreas de estudo na Amazônia Central, indicando os sitios de coletas próximos ao município de Manaus: a - Rio Solimões; b - Lago Central. 


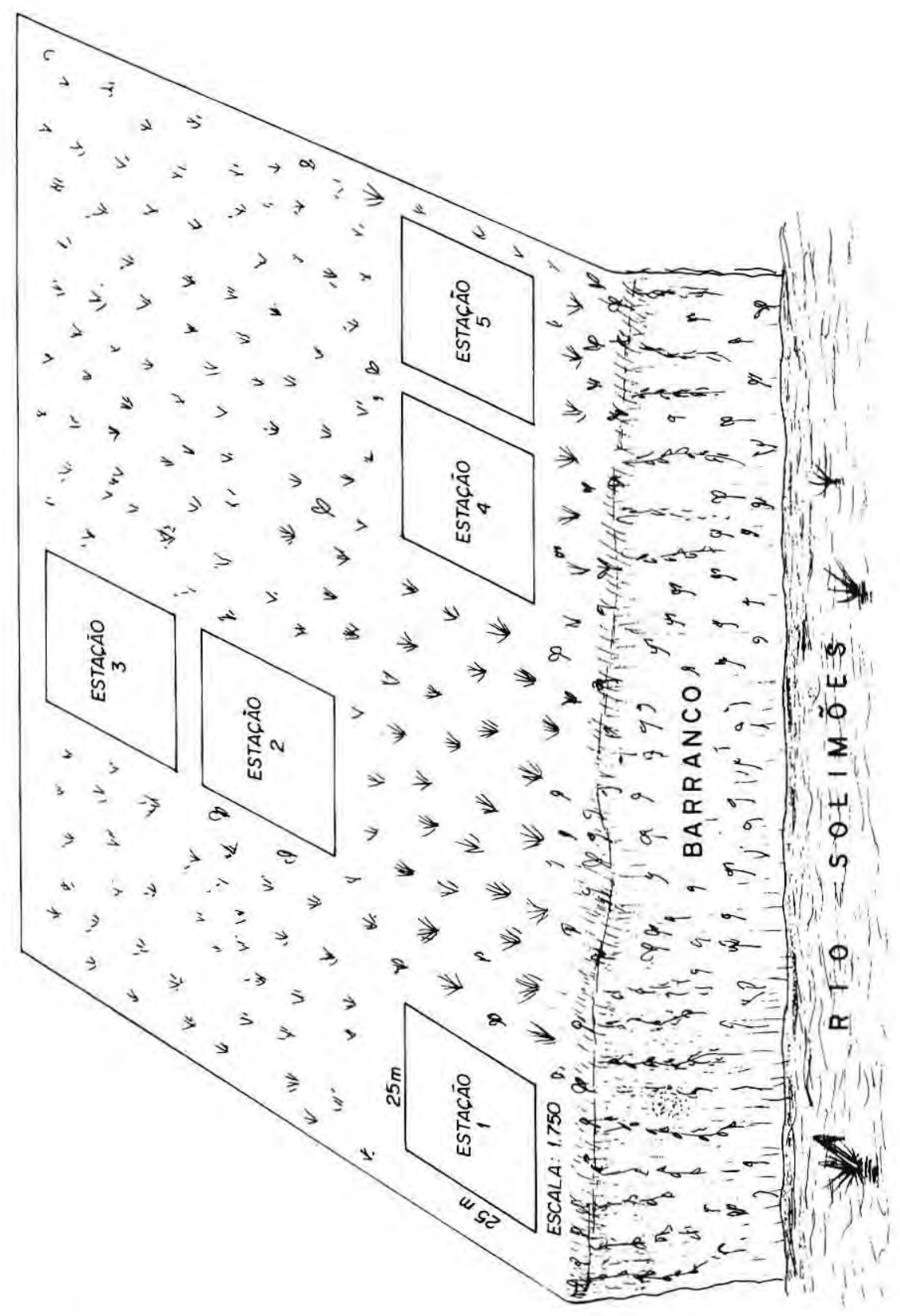

Figura 2. Desenho esquemático da área de coletas do Rio Solimões, indicando a distribuição espacial das cinco estações amostrais. 
área mais larga, em relação ao eixo perpendicular a margem do rio a uma distância de aproximadamente $10 \mathrm{~m}$ do barranco. Isso permitiu a divisão desse sítio em cinco estações de 625 $\mathrm{m}^{2}$, distantes entre si cerca de $60 \mathrm{~m}$. No sítio do Lago Central o relevo era mais acidentado, em uma faixa de terra menos larga, parcialmente ilhada e isolada. Dadas as caracteristicas deste sítio, nele somente foram delimitadas duas estações, cada uma das quais com $1562,5 \mathrm{~m}^{2}$, perfazendo a mesma área total para ambos os sitios amostrais.

\section{MATERIAL E MÉTODOS}

Para a determinação do crescimento de Paspalum fasciculatum, em cada sitio amostral, dez plantas individuais foram marcadas, com fita plástica colorida numerada e amarrada na base do colmo. No sítio do Solimões duas plantas em cada uma das estações de coleta, e no sitio do Lago Central, cinco em cada estação. Esses número amostral foi uma adaptação da metodologia de Piedade (1988) e Rubim (1995) e mostrou-se adequado em testes preliminares que precederam o estudo. O crescimento das plantas foi acompanhado de setembro de 1995 a agosto de 1996 .

Mensalmente, em cada planta marcada foi medido o comprimento do talo, desde a base da planta, rente ao solo, até o ápice, na inserção do último nó. Foi também registrado o número de folhas vivas e mortas, número de nós e entre-nós, bem como observações sobre o número de brotos, ramificações, presença de inflorescência, presença de nós com raízes e sinais de herbivoria. Plantas que acidentalmente foram quebradas ou cortadas foram substituídas por outras de igual tamanho, de forma a ser sempre mantido o mesmo número amostral (Piedade, 1988; Rubim, 1995).

Nos meses correspondentes a fase aquática (março a julho), o comprimento do talo foi medido apenas a partir da superfície da água, tendo sido os valores somados àqueles referentes à profundidade da coluna de água. Como a velocidade e movimentação da água impediram o posicionamento ereto da planta, esta medida foi corrigida pelo valor da diferença entre a altura e o comprimento do talo, obtida em cem plantas medidas durante a fase terrestre.

Os dados foram plotados em planilhas do Microsoft EXCEL versão 7.0. Para verificar se os dados apresentavam uma distribuição normal foram realizados testes de Kolmogorov utilizando-se o Software S.A.S. Quando os dados não foram normais utilizamos teste de estatística não paramétrica como o teste de Willcoxon para a comparação das médias que indicou onde estavam as diferenças e se estas foram significativas para comparações entre duas variáveis (Conover, 1980; Lehman, 1975; Hollander \& Wolfe, 1973).

\section{RESULTADOS Ciclo de vida}

As observações sobre o ciclo de vida de $P$. fasciculatum tiveram inicio em junho de 1995, no pico da fase aquática, quando alguns talos ainda permaneciam emersos, com poucas 


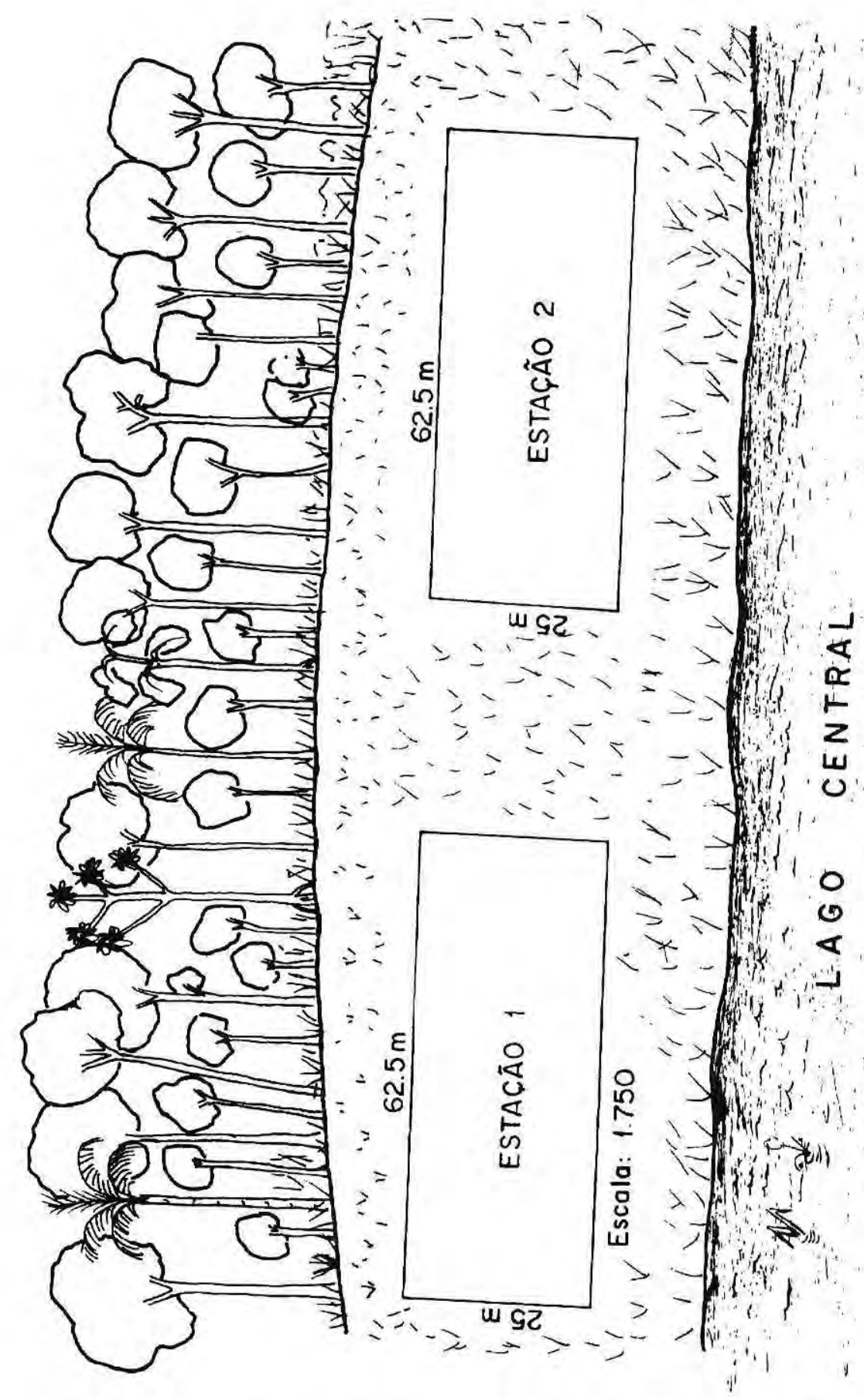

Figura 3. Desenho esquemático da área de coletas do Lago Central, indicando a distribuição espacial das duas estações amostrais. 
folhas. À medida que o nivel da água começou a descer, a partir de agosto, os primeiros talos submersos começaram a aparecer, e neles surgiram novos brotos. Em talos de 90 $\mathrm{cm}$ acima da coluna de água, o número de brotos alcançou o valor máximo de 9 (Tab. 1). Quando os sedimentos foram expostos, os talos, anteriormente submersos, formaram um grande número de brotos. Nos casos em que os talos estavam prostrados rente a superfície do solo, os jovens brotos rapidamente enraizaram, formando novas plantas.

Durante o primeiro mês de observações, setembro de 1995 , o comprimento médio do talo foi de $1,70 \mathrm{~m}$ para as plantas do sitio do Solimões e de $0,62 \mathrm{~m}$ para as do Lago Central (Tab. 1). No período em que as plantas atingiram o maior tamanho, 4,28 m, o número médio de entrenós por planta foi de 44 para as plantas do Solimões e de 77 para as do Lago Central (Tab. 1). Foi formada apenas uma folha em cada nó, tendo a média mensal de folhas verdes por individuo aumentado nos quatro primeiros meses de observações (setembro de 1995 a dezembro de $1995)$, de 18 para 20 , nas plantas do sítio amostral do Solimões, e de 11 para 20, no sítio do Lago Central. Após esse período, em virtude da rápida subida do nivel do rio, inundando os grupamentos de plantas, houve uma redução de $40 \%$ ( 8 folhas remanescentes) no número de folhas do sítio do Solimões, e de $100 \%$ (0 folhas remanescentes) no sitio do Lago Central (Fig. 4). Ao mesmo tempo, o número de folhas mortas aumentou até atingir $100 \%$, quando as águas inundaram completamente as plantas (Fig. 5), uma vez que as folhas, quando submersas, rapidamente amarelaram e apodreceram.

As primeiras inflorescências foram observadas a partir de fevereiro de 1996, no período da enchente, 1 indivíduo florescendo em uma área de $625 \mathrm{~m}^{2}$. O pico máximo de floração foi registrado em abril, 31 indivíduos com inflorescência de 92 plantas contadas em uma área de $10 \mathrm{~m}^{2}$. Entre esses meses, o nivel do rio subiu de $23,39 \mathrm{~m}$ (s.n.m.), em fevereiro, para $26,62 \mathrm{~m}$ (s.n.m.), em abril. Com a submersão e o apodrecimento das folhas nos nós, nestes foram formadas raízes adventícias. O periodo total de submersão dos talos foi de 6 meses, até agosto de 1996, quando o nível da água voltou a descer, recomeçando um novo ciclo.

Durante o ciclo completo de vida acompanhado para a espécie neste estudo, desde o estabelecimento do experimento até a inundação, uma planta individual apresentou o valor máximo de comprimento do talo de 5,05 m (Tab.1). Contudo, em observações aleatórias feitas ao longo de transectos de $50 \mathrm{~m}$, paralelos a margem do rio, foram encontradas plantas com talos medindo até 7,04 m.

\section{Crescimento}

As plantas de $P$ fasciculatum, mostraram um incremento médio no crescimento nos primeiros trinta dias, de $0,57 \mathrm{~m}$, nas estações do Rio Solimões, e de $0,58 \mathrm{~m}$, nas do Lago Central (Fig. 6). Este incremento sofreu redução até um mínimo de 0,24 
Tabela 1. Valores médios mensais dos dados de biometria por plantas individuais de Pfasciculatum $(n=10)$, em regiões de Várzea próximas de Manaus (AM).

\begin{tabular}{|c|c|c|c|c|c|c|c|c|}
\hline \multicolumn{5}{|c|}{ Solimões } & \multicolumn{4}{|c|}{ Lago Central } \\
\hline Mès/ano & $\begin{array}{l}\text { Comp. do } \\
\text { talo (m) }\end{array}$ & Entrenós & Ramificaçס̄es & Brotas & $\begin{array}{l}\text { Comp. do } \\
\text { talo (m) }\end{array}$ & Entrenós & Ramificaçōes & Brotos \\
\hline set/95 & 1,70 & 33 & 2 & 6 & 0,62 & 13 & 0 & 0 \\
\hline out $/ 95$ & 2,27 & 42 & 5 & 9 & 1,20 & 21 & 2 & 2 \\
\hline nov/95 & 2,59 & 46 & 4 & 7 & 1,99 & 30 & 1 & 8 \\
\hline dez/95 & 2,91 & 52 & 3 & 7 & 2,49 & 31 & 1 & 8 \\
\hline $\mathrm{jan} / 96$ & 3,37 & 52 & 2 & 5 & 2,99 & 39 & 0 & 5 \\
\hline fev/96 & 3,90 & 64 & 2 & 7 & 3,52 & 44 & 0 & 7 \\
\hline $\mathrm{mar} / 96$ & 4,14 & 72 & 6 & 8 & 3,86 & 16 & 1 & 7 \\
\hline $\mathrm{abr} / 96$ & 4,53 & 77 & 4. & 7 & 4,28 & 10 & 0 & 0 \\
\hline $\mathrm{mai} / 96$ & 4,21 & 30 & 0 & - & - & - & - & - \\
\hline jun/96 & 4,14 & 20 & 0 & - & - & - & - & - \\
\hline $\mathrm{jul} / 96$ & 3,72 & 24 & 0 & - & - & - & - & - \\
\hline ago/96 & 4,31 & 34 & 0 & 15 & 5,05 & 32 & 0 & 11 \\
\hline
\end{tabular}

Obs.: - Presença de uma coluna de água de $5,20 \mathrm{~m}$ de profundidade, o que impossibilitou a coleta de dados.

m e $0,34 \mathrm{~m}$, em março de 1996, nas áreas do Solimões e do Lago Central, respectivamente. Nos meses correspondentes a fase aquática (maio, junho e julho), houve um incremento negativo para as plantas do sítio do Rio Solimões, enquanto que as plantas do Lago Central não puderam ser localizadas. Contudo, no mês de agosto, quando a água do rio começou a descer, foram registrados para $P$. fasciculatum, valores de incremento médio de $0,59 \mathrm{~m}$ e $0,77 \mathrm{~m}$, para as plantas das áreas do Rio Solimões e do Lago Central, respectivamente (Fig. 6). Considerando a média total do comprimento dos talos, verificou-se que as plantas do Rio Solimões apresentaram os maiores comprimentos, $0,75 \mathrm{~m}$ acima daqueles registrados para as do Lago Central. Esta diferença é significativa $(\mathrm{p}<0,05)$, isto é , estatisticamente as plantas da área do Solimões registraram maior taxa de crescimento (Fig. 7).

\section{DISCUSSÃO}

Nas áreas de várzea da Amazônia Central, a distribuição da vegetação é influenciada por vários fatores, entre eles, a duração das fases aquáticas e terrestres que, para plantas herbáceas, é de grande importância, 
determinando composição de espécies e hábitos de vida das plantas ao longo do ano (Junk \& Piedade, 1997). Esses mesmo fatores são considerados como de grande, importância para a vegetação da Amazônia Peruana (Puhakka \& Kalliola, 1993).

Paspalum fasciculatum ocorre em grandes densidades, preferencialmente nas regiões mais marginais de rios e lagos, entre as cotas de 23 a $27 \mathrm{~m}$ (s.n.m.), podendo entretanto ocorrer em áreas superiores do gradiente, desde que não haja sombreamento, o que em geral ocorre quando são derrubadas as árvores da floresta alagável. Estas regiões são definidas por Junk et al. (1989), como a zona de transição entre o ambiente aquático e terrestre, e as comunidades que ai habitam possuem adaptações para suportar a instabilidade desses ambientes.

Muitos autores citam certas características como respostas adaptativas das espécies a determinadas condições ambientais. No caso específico dos ambientes enfocados neste estudo, essas características relacionam-se principalmente a adaptações decorrentes da existência de diferentes regimes hidrológicos, tais como, aumento do comprimento da planta durante a fase de inundação, como se verifica para a espécie Echinochloa polystachya, cujo ciclo de vida é sincronizado com a subida do nivel da água e a fase aquática do ciclo hidrológico (Piedade et al., 1992; Piedade, 1993), capacidade de rápida recolonização de muitos habitats dentro de um gradiente de planícies inundáveis, formação de raizes adventícias e predominância de propagação vegetativa, entre outras (Junk, 1970; Fittkau et al., 1975; Junk \& HowardWilliams, 1984; Loreti \& Oesterheld, 1996; Junk \& Piedade, 1997).

Dentre as caracteristicas citadas como adaptativas à instabilidade dos ambientes de áreas alagáveis, $P$. fasciculatum apresenta três bem marcantes. A espécie ocorre em grandes densidades, ocupando áreas com mais de $500 \mathrm{~m}^{2}$, em grupamentos monospecíficos. Quando em presença de outras espécies que não a sombreiem, ela torna-se dominante, apresentando sempre maior densidade que as outras (Conserva \& Piedade, 1998). Além disso, as populações observadas ocorreram em uma faixa relativamente ampla de cotas no gradiente de inundação, de 23 a 27 m (s.n.m.).

Embora quando ocorrendo nas cotas mais baixas, $P$. fasciculatum ocupe as mesmas áreas que Echinochloa polystachya, a primeira tem seu ciclo de vida sincronizado com a fase terrestre do ciclo hidrológico. Ainda que os maiores valores de incremento no comprimento dos talos tenham sido observados no final da descida das águas e na fase terrestre, a grande adaptação da planta aos ambientes inundáveis é demonstrada pela grande tolerância de seus talos à submersão por longos períodos. Junk \& HowardWilliams (1984), citam que a espécie pode suportar de dois a três anos de inundação contínua, o que a leva a ser classificada freqüentemente como planta semi-aquática (Fittkau et al., 1975; Piedade et al., 1992).

Os valores totais de 


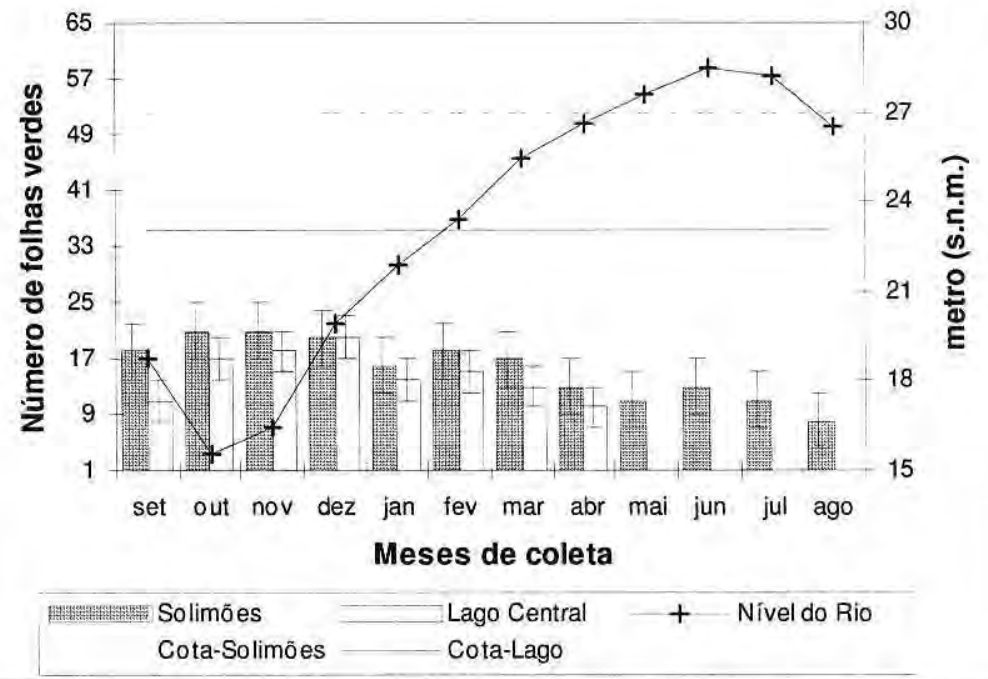

Figura 4. Variação mensal da média e desvio padrão do número de folhas verdes por indivíduo de Paspalum fasciculatum, nas áreas do Solimões e do Lago Central, durante o periodo de estudo (de set/95 a ago/96), em relação a flutuação do nível da água (dados da CODOMAR).

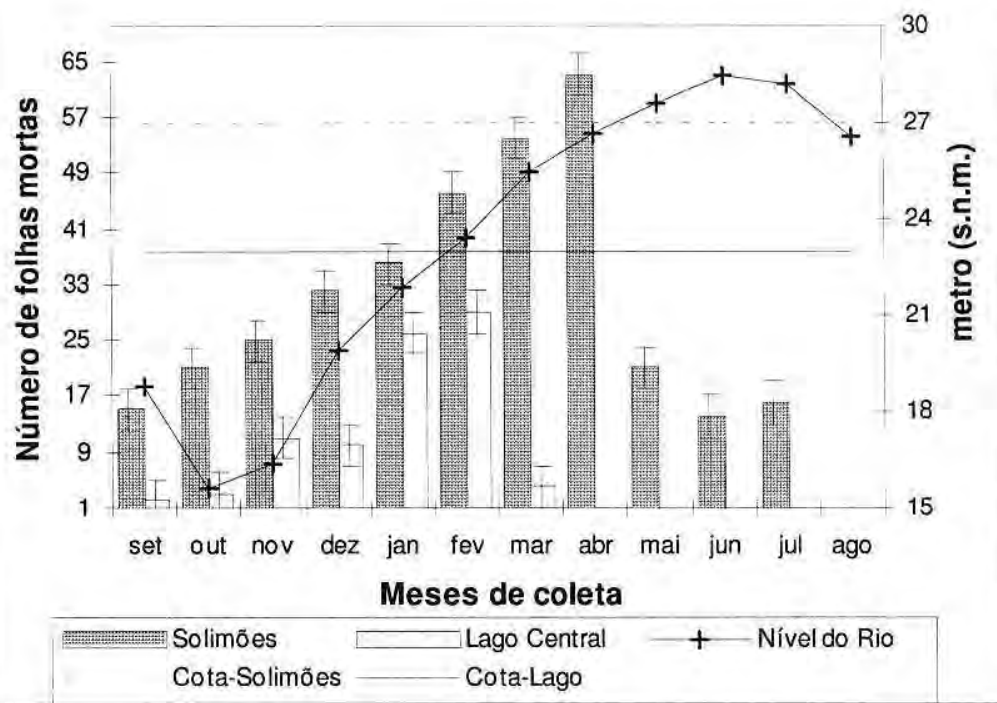

Figura 5. Variação mensal da média e desvio padrão do número de folhas mortas por indivíduo de Paspalum fasciculatum, nas áreas do Solimões e do Lago Central, durante o período de estudo (de set/95 a ago/96), em relação a flutuação do nivel da água (dados da CODOMAR). 


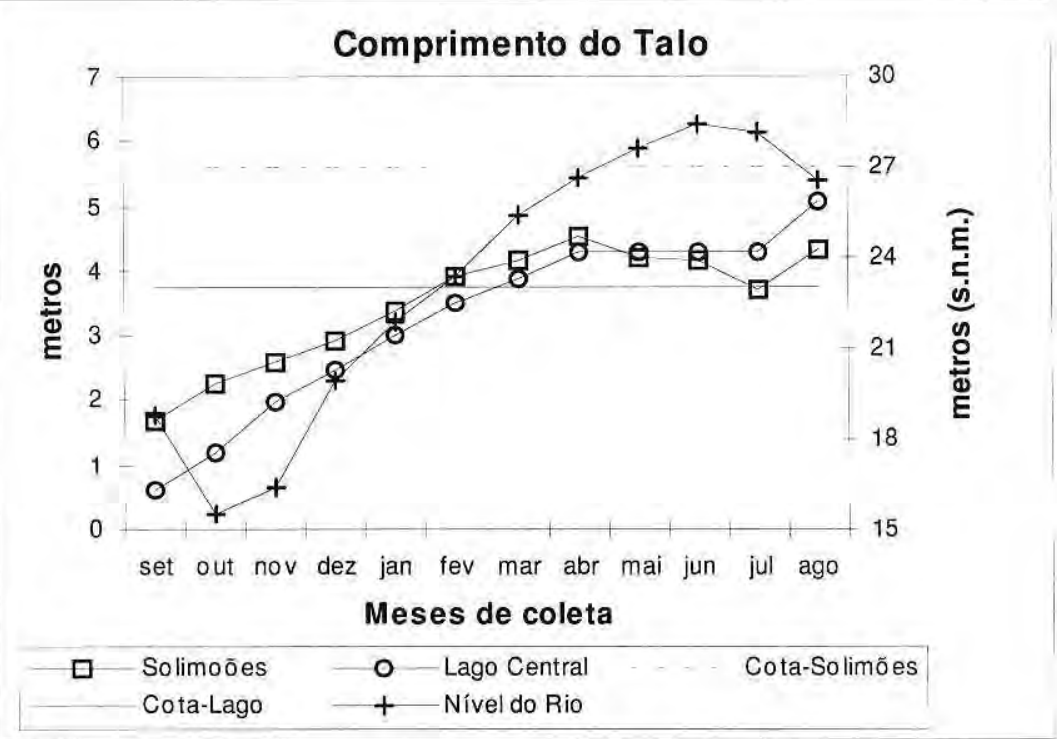

Figura 6. Variação mensal da média do comprimento do talo de Paspalum fasciculatum, das populações do Solimòes e do Lago Central, em relação a flutuação do nivel da água (dados da CODOMAR).

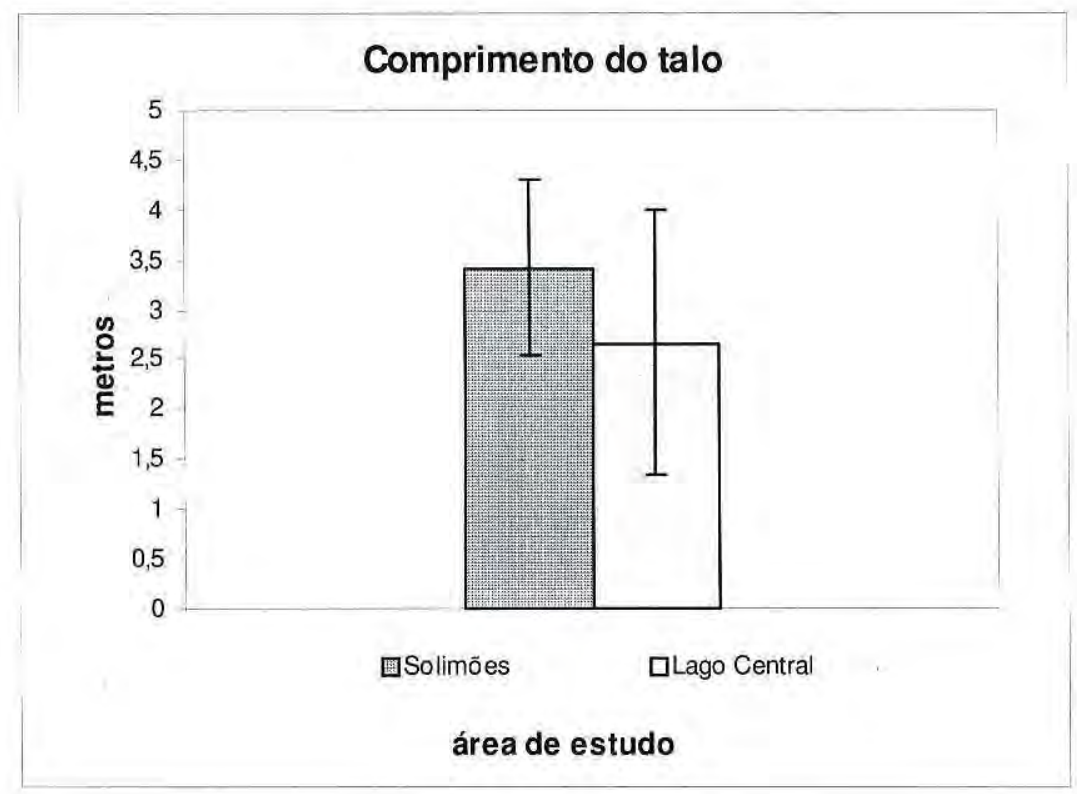

Figura 7. Comparação da média total $( \pm 1,31)$ do comprimento do talo de Paspalum fasciculatum, das populações do Solimões e do Lago Central. 
comprimento registrados diferiram entre os dois sitios amostrais, com 3,41 $\mathrm{m}$ e 2,66 $\mathrm{m}$ para as áreas do Rio Solimões e do Lago Central, respectivamente. Essa variação pode ser decorrente da diferença entre as cotas de ocorrência existente entre os dois sítios. O sítio amostral do Lago Central situou-se na cota de $23 \mathrm{~m}$ (s.n.m.), tendo sido a área submetida a duas inundações sucessivas próximo ou durante o periodo de estudo (quatro meses - abril a agosto de 1995, antes do periodo de estudo; seis meses fevereiro a agosto de 1996, durante o periodo de estudo), com um intervalo de seis meses entre elas. Por outro lado, a população do Rio Solimões, por estar situada em uma cota mais alta, 27 m (s.n.m.), foi submetida, no mesmo intervalo, a um período de inundação de apenas três meses, o que pode explicar o maior crescimento, devido a maior duração da fase terrestre (Junk \& Piedade, 1997). Contudo, apesar dessas diferenças, após o período de inundação, em ambas as áreas as plantas retomaram o crescimento, apresentando valores de incremento de $0,59 \mathrm{~m}$ e $0,77 \mathrm{~m}$ nas áreas do Solimões e Lago Central, respectivamente, superiores àqueles obtidos no início do estudo.

$\mathrm{O}$ aumento no número de folhas verdes nos quatro primeiros meses de estudo foi superior na área do Lago Central $(45 \%)$, que na área do Rio Solimões (14\%), o que é compativel com a maior retenção de nutrientes esperada em um lago de várzea, em relação ao sistema maior de drenagem (Furch, 1984). A quantidade de folhas mortas, que chegou a $100 \%$ com a subida do nivel da água e que permanece no solo quando a água retorna a seu nivel normal iniciando a fase terrestre, pode desempenhar um importante papel na manutenção dos níveis de nutrientes e umidade do solo, uma vez que a categoria das folhas verdes é a que apresenta a maior concentração de nutrientes, e valores de biomassa, representando mais de $15 \%$ da biomassa vegetal viva (em média 3,2 t/ha), em ambos os sitios amostrais (Conserva, 1999), confirmando a importância já sugerida para a espécie no balanço nutricional das várzeas (Piedade et al., 1991; Piedade et al., 1994). Para as espécies Echinochloa polystachya (Piedade et al., 1997) e Oryza spp. (Rubim, 1995), devido a intensa decomposição durante a fase terrestre, os talos, embora não tenham os mais altos teores de nutrientes, em virtude de sua elevada biomassa, são a categoria da planta mais importante. Contudo, no caso de Paspalum fasciculatum, ao contrário das espécies anteriormente citadas, grande parte dos talos permanece viva, mesmo após a sedimentação e formação de novos brotos.

Embora o número de indivíduos produzindo inflorescências seja pequeno em relação as densidades da planta (em média 21 indivíduos por $\mathrm{m}^{2}$ e apenas 4 com inflorescências), a reprodução sexuada deve desempenhar um importante papel para a colonização de novos ambientes pela espécie, já que as sementes são produzidas no período de águas altas, o que aumenta a amplitude de 
distribuição através da dispersão pela água. Entretanto, durante este estudo foi possível verificar também a forte predominância da propagação vegetativa, o que é interpretado como uma estratégia para compensar as altas taxas de perda de biomassa, verificadas durante os períodos menos favoráveis do ciclo hidrológico (Junk \& Piedade, 1997). Muitas espécies não se manteriam exclusivamente através da reprodução sexuada, uma vez que as condições para a germinação de sementes e esporos muito raramente são obtidas em ambientes alagáveis (Junk, 1970; Piedade, 1988; Oliveira, 1992; Oliveira, 1998).

O ciclo de vida e a ecologia de Paspalum fasciculatum demonstram uma estreita adaptação da espécie à instabilidade dos ambientes de várzea, especialmente da região da Amazônia Central, com grandes amplitudes e eventos catastróficos freqüentes. A espécie tolera as rápidas mudanças do ambiente físico, podendo ser considerada um bom exemplo de planta representativa dos primeiros estágios de colonização das margens sedimentares de rios e lagos. A dormência dos talos e a grande tolerância a anos subsequentes de inundação apontam a espécie como uma estrategista "r" (Pianka, 1970), altamente adaptada às áreas alagáveis de elevado status nutricional.

\section{AGRADECIMENTOS}

Este estudo foi possível graças ao financiamento do projeto INPA/Maxplanck e programa SHIFT de cooperação Brasil/Alemanha. O CNPq forneceu bolsa de estudos a primeira autora. Agradecemos aos Técnicos Valdeney de Araújo Azevedo e Celso Rabelo da Costa, bem como a todo pessoal de apoio do BADPI, pela ajuda no trabalho de campo.

\section{Bibliografia citada}

Conover, W. J. 1980. Pratical nom parametric statistcs 2 nd. Edition, John Willey and Sons, Inc. New York. 423 p.

Conserva, A. S 1999. Biomassa, Ciclo de vida e Composição Quimica de duas populações de Paspalum fasciculatum Willd ex. Fluegge (Poaceae) em diferentes habitats de Várzea da Amazonia Central. Dissertação de mestrado, Instituto Nacional de Pesquisas da Amazônia/ Fundação Universidade do Amazonas. Manaus, Amazonas. 87p.

Conserva, A. S.; Piedade, M. T. F. 1998. Influence of flood-pulse and land-use on the composition of herbaceous species on a floodplain in Central Amazonia. Verh. Internat. Verein. Limnol. Volume 26. Pgs. 994 - 995. Stuttgart, março de 1998.

Cook, C.D.K.; Gut, B.J.; Rix, E. M.; Schneller, J.; Seitz, M., 1974 Water Plants of the World. W. Junk publ. 561p.

Fittkau, E. J.; Irmler, U.; Junk, W. J.; Reiss, F.; Schmidt, G. W. 1975. Productivity, Biomass, and Population Dynamics in Amazonian Water Bodies. In: Galey, Z.B.; Medira, B, (eds). Amazonian Aquatic Systems: by Springer-Verlag New York Inc. 289-311.

Furch, K. 1984, Seasonal variation of the major cation content of the várzea-lake Lago Camaleão, middle Amazon, Brazil, in 1981 and 1982. Verh. Internat. Verein. Limnol. 22: 1288-1293.

Furch, K.; Junk, W. J. 1997. Physicochemical Conditions in the floodplains. In: Junk W.J.(ed.) Ecological Studies. The Central Amazon Floodplain Springer - Verlag Berlin Heidelberg. 126: $69-108$.

Hollander, M,; Wolfe, D. A. 1973. Non parametrics statistical metods. John Wiley 
and sons. New York. $458 \mathrm{p}$.

Howard-Williams, C.; Junk, W. J. 1976. The Decomposition of Aquatic Macrophytes in the Floating Meadows of a Central Amazonian Várzea Lake. Biogeographica vol. 7: 115-123.

Howard-Williams, C.; Junk, W. J. 1977. The Chemical Composition of Central Amazon Aquatic Macrophytes with Special Reference to their Role in the Ecosystem. Arch. Hydrobiol. vol. 79 (4): 446-464.

Index Kewensis. 1895. Plantarum Phanerogamum. América Tropical. pp 430.

Junk, W. J, 1970. Investigations on the Ecology and Production-Biology of the "Floating Meadows" (Paspalo-Echinochloetum) on the Middle Amazon. Part I: The Floating Vegetation and its Ecology. Amazoniana vol. II (4): 449-495.

Junk, W. J. 1993. Wetlands of Tropical South America. In: Whigham, D., Hejny, S. \& Dykyjove, D. (eds.): Wetlands of the World. Dr. W. Junk Publ., Dordrecht, Boston, Lancaster: 679-739

Junk, W, J.; Howard-Williams, C. 1984. Ecology of Aquatic Macrophytes in Amazonia. In: Sioli, H. (ed.). The Amazon: limnology of a mighty tropical river and its basin. W. Junk Publ., Dordrecht: 269-293 pp.

Junk, W. J.; Bayley, P. B.; Sparks, R. E. 1989. The flood pulse concept in river-floodplain systems, In: Dodge, D, P. (ed.). Proceedings of the International Large River Symposium (LARS). Can. Spec. Publ. Fish. aquat. Sci. 106: 110-127.

Junk, W. J.; Piedade, M. T. F. 1993a. Herbaceous plants of the Amazon floodplain near Manaus: Species diversity and adaptations to the flood pulse. Amazoniana XII (3/4): 467-484.

Junk, W. J.; Piedade, M. T. F. 1993b. Biomass and primary-production of herbaceous plants communities in the Amazon floodplain. Hydrobiologia 263: 155-162.

Junk, W. J.; Piedade, M. T, F. 1997. Plant life in the floodplain with special reference to herbaceous plants. In: Junk, W.J. (ed.) Ecological Studies, The Central amazon
Floodplain. Springer-Verlag Berlin Heidelberg. 126: 147-185.

Lehman, E. L. 1975. Non parametrics: statistical methods based on rank. San Francisco: Holden-Day, Inc. 238 p.

Loreti, J.; Oesterheld, M. 1996. Intraespecifc variation in the resistance to flooding and drought in populations of Paspalum dilatatum from different topografic positions. Oecologia 108: 279-284.

Martius, C. K. F. 1842. Flora Brasiliensis. Verlag J. Cramer, New York, NY. vol. II. $118 \mathrm{p}$.

Oliveira, A. C. 1998. Aspectos da dinâmica populacional de Salix martiana Leyb. (Salicaceae) em áreas de várzea da Amazônia Central. Dissertação de mestrado, Instituto Nacional de Pesquisas da Amazônia/ Fundação Universidade do Amazonas. Manaus, Amazonas. 103p.

Oliveira, G. C. X. 1992. Padrões de variação fenotipica e ecologia de Oryzae (Poaceae) selvagens da Amazônia. PPg, ESALQ, dissertação de mestrado. São Paulo. 303p.

Pianka, E. R. 1970. On r and K - selection. $A M$. Nat. 104: 592-597.

Piedade, M. T. F. 1988. Biomassa, produtividade e atividade fotossintética de Echinochloa polystachya (H.B.K.) Hitchcock (Gramineae $=$ Poaceae $)$ capim semi-aquático da Várzea Amazônica. Tese de doutorado INPA/FUA. $154 \mathrm{p}$.

Piedade, M. T. F. 1993. Biologia e ecologia de Echinocloa polystachya (H. B. K.) Hitchock $($ Gramineae $=$ Poaceae $)$, capim semi-aquático da várzea amazônica Acta Limnol. Bras. VI: 173-185.

Piedade, M. T. F.; Junk W. J.; Long. S. P. 1991. The productivity of the $\mathrm{C}_{4}$ grass Echinochloa polystachya on the amazon floodplain. Ecology 72 (4), 1456 - 1467.

Piedade, M. T. F.; Junk, W. J.; Long, S. P. 1997. Nutrient dynamics of the highly productive $\mathrm{C}_{4}$ macrophyte Echinochloa polystachya on the Amazon floodplain. Functional Ecology. 11: 60-65.

Piedade, M. T. F.; Junk W. J.; Mello, J. A. N. 
1992. A floodplain grassland of the central Amazon. In: Long, S.P.; Jones, M.B.; Roberts, M.J. (eds). Primary Productivity of Grass Ecossystems of the Tropics and Sub-tropics. Chapmam \& Hall. London. 127-158.

Piedade, M. T. F.; Long, S. P.; Junk, W. J. 1994. Leaf and canopy uptake of stand of Echinochloa polystachya on the Central Amazon floodplain. Oecologia. 97: $159-$ 174.

Prance, G. T. 1979. Notes on the vegetation of Amazonia III. The terminology of amazonian forest types subject to inundation. Brittonia. 31: 26-38.

Puhakka, M.; Kalliola, R. 1993. La vegetación en áreas de inundación en la selva baja de la Amazonia peruana (cap. 8). In: Kalliola, Ppuhakka, Danjoy (Eds.) Amazonia Peruana: Vegetación húmeda tropical en el llano subandino. Proyecto Amazonia, Universidad de Turku. 113 - 138.

Rubim, M. A. L. 1995 Ciclo de vida, biomassa, e composiçào química de duas espécies de arroz silvestre da Amazônia Central. Dissertação de mestrado, Instituto Nacional de Pesquisas da Amazônia/ Fundação Universidade do Amazonas. Manaus, Amazonas. 126 p.

Schimidt, W. G. 1973. Primary production of phytoplanckton in the three types of Amazonian waters II. The limnology of a tropical floodplain lake in Central Amazonia Lago do Castanho. Amazoniana, 4:139203.

Sioli, H. 1975. Tropical rivers as expressions of their terrestrial environments. $I n$ : Golley, F.B.; Medina, E. (eds) Tropical ecological systems. Trends in terrestrial and aquatic research. Spriger, Berlin. 275-288.
Sioli, H. 1984. The Amazon and its main affluents: Hydrography, morphology of the river courses, and river types. $I n$ : Sioli, $\mathrm{H}$. (ed) The Amazon Limnology and landscape ecology of a mighty tropical river and its basin. W. Junk Publishers. Netherlands. $127-165$.

Sioli, H. 1985. Amazônia fundamentos da ecologia da maior região de florestas tropicais. Ed. Vozes. Petrópolis, RJ. 72 p. 